\title{
A mechanical model and stress-strain response of the biceps brachii under static load
}

\author{
Hamid Asadi Dereshgi', Kasım Serbest ${ }^{2 *}$, Sema Nur Şahin' ${ }^{1}$, Büşra Balık ${ }^{1}$ \\ 'Department of Biomedical Engineering, Istanbul Arel University, Istanbul, Turkey, ${ }^{2}$ Department of Mechatronics Engineering, Sakarya University of \\ Applied Sciences, Sakarya, Turkey
}

Orcid: H. Asadi Dereshgi (0000-0002-8500-6625), K. Serbest (0000-0002-0064-4020), S.N. Şahin (0000-0003-3549-7646), B. Balık (0000-000I-7388-6605)

\begin{abstract}
Muscle contraction is a complex phenomenon that begins with chemical processes, continues physiologically, and leads to the production of force. Although the production of force in the muscles depends on factors such as temperature, age, gender, race, but the most important factor is the external load applied to the muscle. Determining the effects of increased load on muscle mechanics is of particular importance for planning exercise activities and rehabilitation processes. In this study, the effects of different external forces on the stress and pressure behavior of the muscle were examined on a simplified model of the biceps. Accordingly, a finite element model of the biceps brachii muscle fiber was constructed. The application of different static loads $(2.5-100 \mathrm{~N})$ on both the proximal tendon (one-directional) and the proximal and distal tendon (bidirectional) together were investigated. According to the results, it was found that the external force applied in both directions causes a significant increase in displacement behavior and stress.
\end{abstract}

Keywords: Finite element model, Link-segment model, Muscle fiber, COMSOL Multiphysics.

\section{Intorduction}

The muscular system, forming $40-45 \%$ of the body weight and together with the skeletal system provides the forces needed to perform the movement. Musculoskeletal system consisting of bone, cartilage, muscle, tendon and connective tissue; Skeletal muscles consisting of muscle fibers with 10 to 100 micrometers thick and 1 to $30 \mathrm{~cm}$ long, they were placed as a layers on the bones and were connected to the bones by tendons $[1,2]$. Contractions in muscle fiber cause muscles to exhibit complex mechanical properties as a result of nerve stimulation [3,4]. Hill and Huxley's models provide mathematical notations used to analyze these complex mechanical properties [5-8]. The Hill muscle model, which consists of three elements: the active contractile element, the serial elastic element and the parallel elastic element, reflects inactive features, mainly used to explain the behavior of the muscle and tendon $[9,10]$. Therefore, it has been widely applied in simulations of human movement [11-13] and is still applied in multi-body simulations [14-16].

There are other muscles around the muscles in the human body, bones and connective tissue. When external forces were transmitted to the muscle, the strength and properties of muscle strength were affected $[17,18]$. More accurate results in the developed muscle models could only be obtained by considering this effect. Since explaining the relationships between length, force and speed was important in determining muscle characteristics, various studies had been performed on muscle-to-muscle fibers [19-24].

Here are some studies from the past to the present to model and simulate muscle contraction under different conditions: Liber (1993) investigated the possibility of examining surgical methods including skeletal muscle by considering the muscles and the main function of the muscles [25]. Shue and Crago (1998) developed a nonlinear parameter estimation algorithm with the Hill type muscle-tendon models, they additionally determined the static parameters and behavior of the muscle during random stimulation [26]. Donkelaar et al. (1999) investigated the two-dimensional (transverse and longitudinal) surface deformation and stretching of skeletal muscle by fabricating three-dimensional measurements at different muscle lengths [27]. Stäubli et al. (1999) analyzed the mechanical tensile properties of quadriceps tendons and patellar ligaments using the harvesting technique [28]. Using a musculoskeletal modeling package to simulate musculoskeletal surgery, Holzbauer et al. (2005) developed a three-dimensional model that describes mechanical movement that included all major upper limb muscles
*Corresponding author.

Email: kserbest@subu.edu.tr
European Mechanical Science (2022), 6( I ): 27-3 I

https://doi.org/I0.2670 I/ems. I0I5772

Received: October 28, 2021 - Accepted: January 20, 2022 
[29]. Martinek et al. (2008) simulated Hodgkin-Huxley-like excitation with COMSOL Multiphysics using the bidomain model and examined muscle fiber responses to changes in parameters [30]. Kochbach et al. (2011) performed simulations to determine the optimal parameters and conditions for thermal stimulation of muscle tissue using COMSOL Multiphysics finite element software [31]. Carbon et al. (2016) performed a comprehensive sensitivity analysis by simulating the lower limb model in the AnyBody modeling system to evaluate the effects of possible errors in the parameters of the Hill-type musculoskeletal model on the tendons of the musculoskeletal model [32]. Esmaeili and Maleki (2020) with muscle coordination analysis and time-varying muscle synergy extraction from surface electromyography (sEMG) models, investigated the similarity of muscle synergies for mechanical conditions [33].

Musculoskeletal models in these studies had been developed to model and simulate muscle contractions under the influence of various static forces, these findings play an active role in the treatment of various diseases, human movement analysis and determining the treatment process.

The purpose of this study was to analyze the effect of static forces applied on muscle fiber at regular intervals on muscle mechanics. Changes in displacement and stress due to static forces in the muscle were examined. The results of the analysis help to better understand the effect of changing muscle forces on muscle mechanics and the relationship between contraction and force.

\section{Materials and Methods}

A model of the forearm, upper arm, elbow joint and shoulder joint was performed to simulate the effect of static forces on the muscle fiber (see Figure 1). Static force was applied at force values up to $100 \mathrm{~N}$ and with constant increments of $2.5 \mathrm{~N}$ starting from $2.5 \mathrm{~N}$. According to designing muscles and tendons in the form of cylinders and bones in the form of cubes (see Figure 2), a finite element model of biceps brachii muscle fiber; consisting of bone, muscle fiber and tendon structures was performed. In the model, the length of the bone margin was $100 \mathrm{~mm}$, the length of the muscle fiber was $250 \mathrm{~mm}$, the length of the tendon was $25 \mathrm{~mm}$ and the diameter of the muscle fiber and tendon was $1 \mathrm{~mm}$. Young's modulus of muscle fiber, bone and tendon were $1.162 \times 106 \mathrm{~Pa}, 1.0 \times 1010 \mathrm{~Pa}$ and $1.6 \times 106 \mathrm{~Pa}$, respectively; Poisson's ratio were $0.4,0.3$ and 0.497 , respectively; their densities were $1056 \mathrm{~kg} / \mathrm{m} 3,2570$

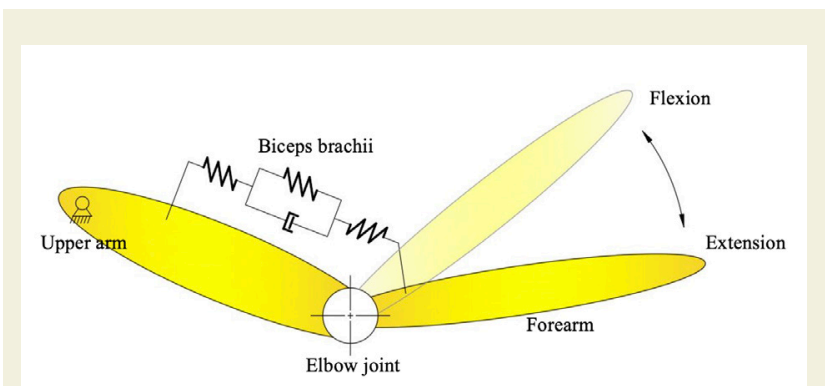

Figure 1. Mechanical model of the biceps brachii with arm.

$\mathrm{kg} / \mathrm{m}^{3}$ and $1670 \mathrm{~kg} / \mathrm{m}^{3}$.

Grid convergence analysis with a sensitivity of 0.001 was used to check the accuracy of the analyzes performed in COMSOL Multiphysics 5.5. Three different grids (see Figure 3), normal, fine and finer, respectively, with the characteristics given in the table were used. The error rate between the first meshing stage and the second meshing stage was $0.10 \%$, while the error rate between the second meshing stage and the meshing stage was $0.28 \%$. In the grid convergence method (see Figure 4), although the large number of mesh elements increased the accuracy of the results, the displacement values were very close in the second and subsequent steps, in order to optimize the analysis time, the fine mesh was preferred (see Table 1).

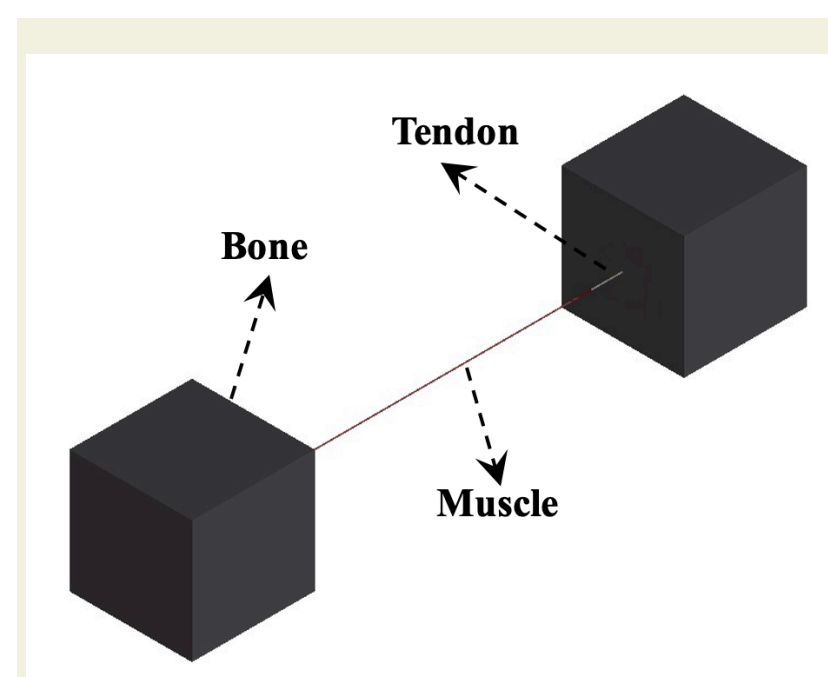

Figure 2. The finite element structure of the muscle, tendon and bone.

The results of the mechanical behavior of the muscle fiber were presented in Chapter 3 by examining parameters such as displacement, strain, and strain. Due to the application of external force only on the proximal tendon (one-directional) and both proximal and distal tendons

\begin{tabular}{|c|c|c|c|c|c|}
\hline $\begin{array}{l}\text { Domain element } \\
\text { statistics }\end{array}$ & Number of elements & $\begin{array}{l}\text { Minimum element } \\
\text { quality }\end{array}$ & $\begin{array}{c}\text { Average element } \\
\text { quality }\end{array}$ & Element volume ratio & Mesh volume $(\mathrm{mm} 3)$ \\
\hline Normal & 1599 & 0.08598 & 0.6013 & 0.00002957 & 2001000 \\
\hline Fine & 2570 & 0.1201 & 0.6034 & $0.0000577 \mid$ & 2001000 \\
\hline Finer & 4559 & 0.2196 & 0.6316 & 0.00003292 & 2001000 \\
\hline
\end{tabular}


(a)

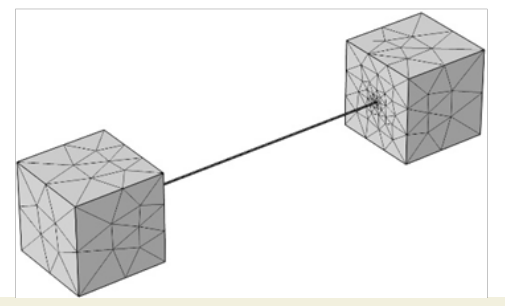

(b)

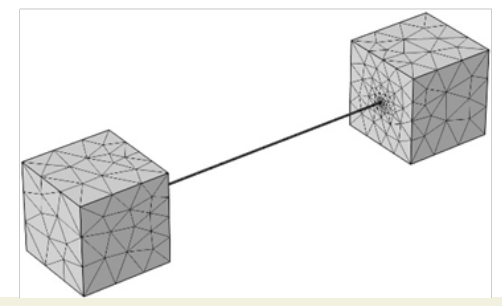

(c)

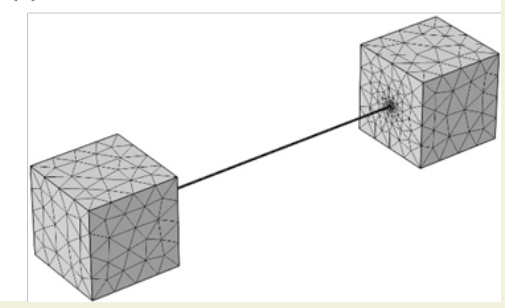

Figure 3. Mesh types, a) Normal, b) Fine and c) Finer.

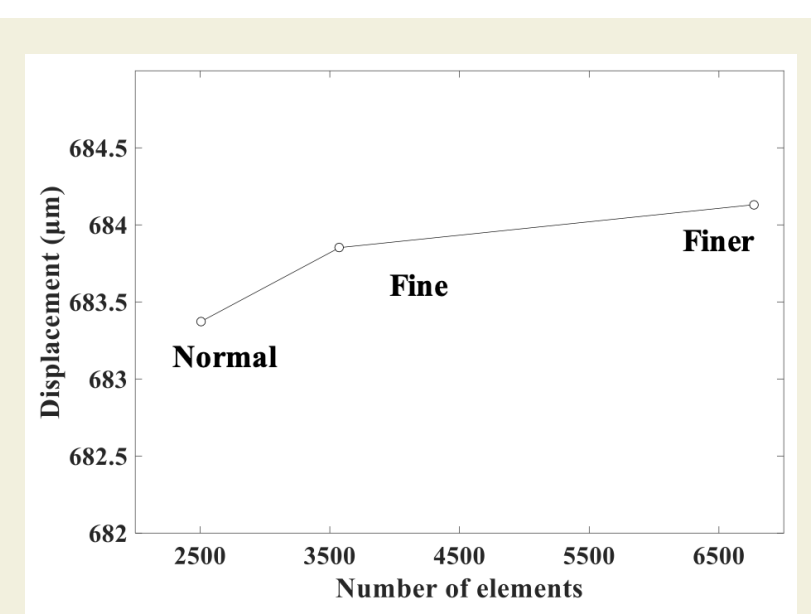

Figure 4. Mesh convergence analysis

(bidirectional) analysis was performed.

\section{Results and Discussions}

The simulation results with finite element analysis are shown in Figure 5. Here are the results of muscle displacement. Figure 6 shows the displacement change caused by the static load applied in both directions. Here, it was understood that the applied static force and the elongation in the muscle were linear. When a maximum force of 100 $\mathrm{N}$ was applied, $6.99 \mathrm{~mm}$ elongation occurs in the biceps brachii fiber.

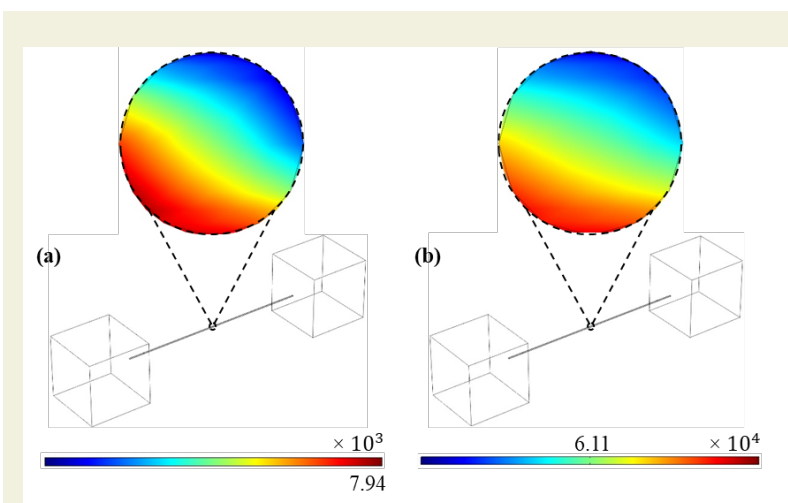

Figure 5. Displacement analysis of the finite element model, a) one-directional b) bidirectional.

The displacement change that occurs when external load was applied only to the proximal tendon. Here, when a maximum force of $100 \mathrm{~N}$ was applied, it was seen that the biceps birachii fiber elongates by $0.2 \mathrm{~mm}$. It was additionally seen that there was a linear relationship between force and displacement in the case of applying a unidirectional force. The coefficient of determination (R2) was calculated as 0.9995 in case of double-sided force application, and 0.9991 in case of unidirectional force application.
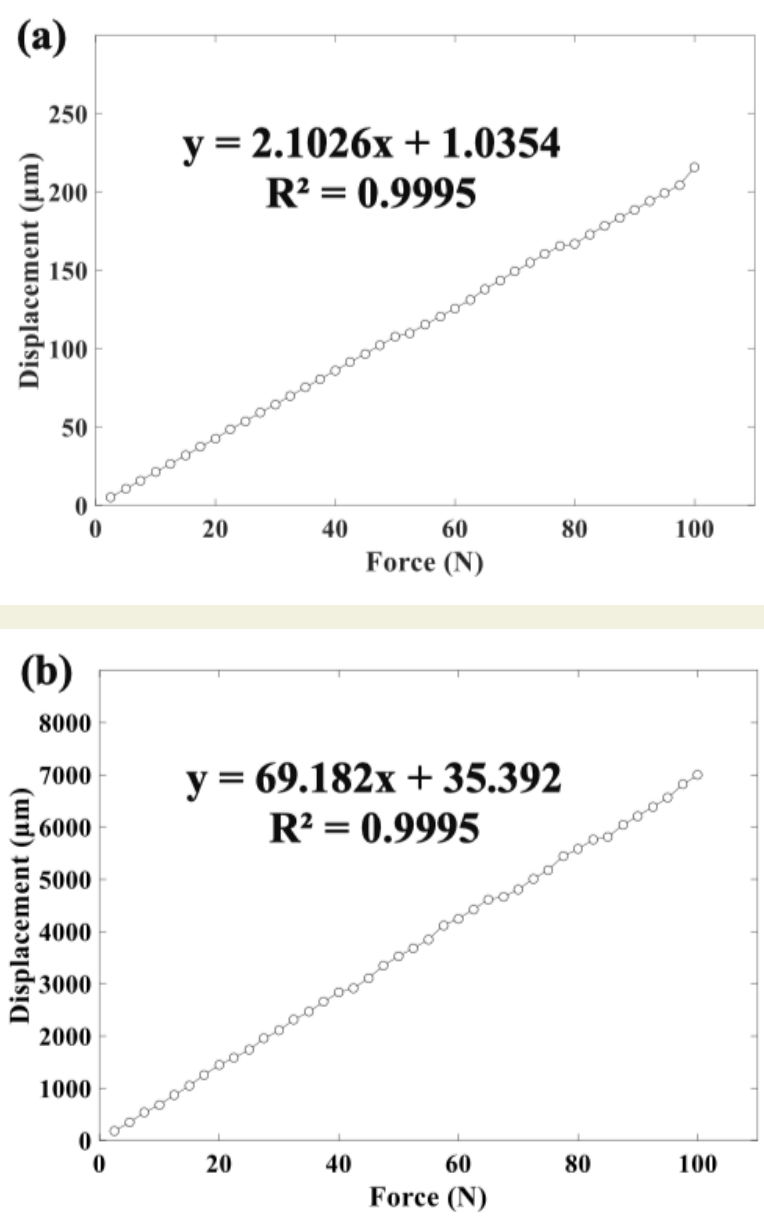

Figure 6. Displacement-force response of the biceps brachii, a) one-directional b) bidirectional.

Figure 7 shows the stress-strain behavior in the muscle when unidirectional and double wool load were applied, respectively. It was understood that there was a linear relationship between stress and strain in both loading conditions. In the case of applying a maximum force of $100 \mathrm{~N}$, $65502 \mathrm{~Pa}$ strain occurs in the muscle fiber in unidirection- 

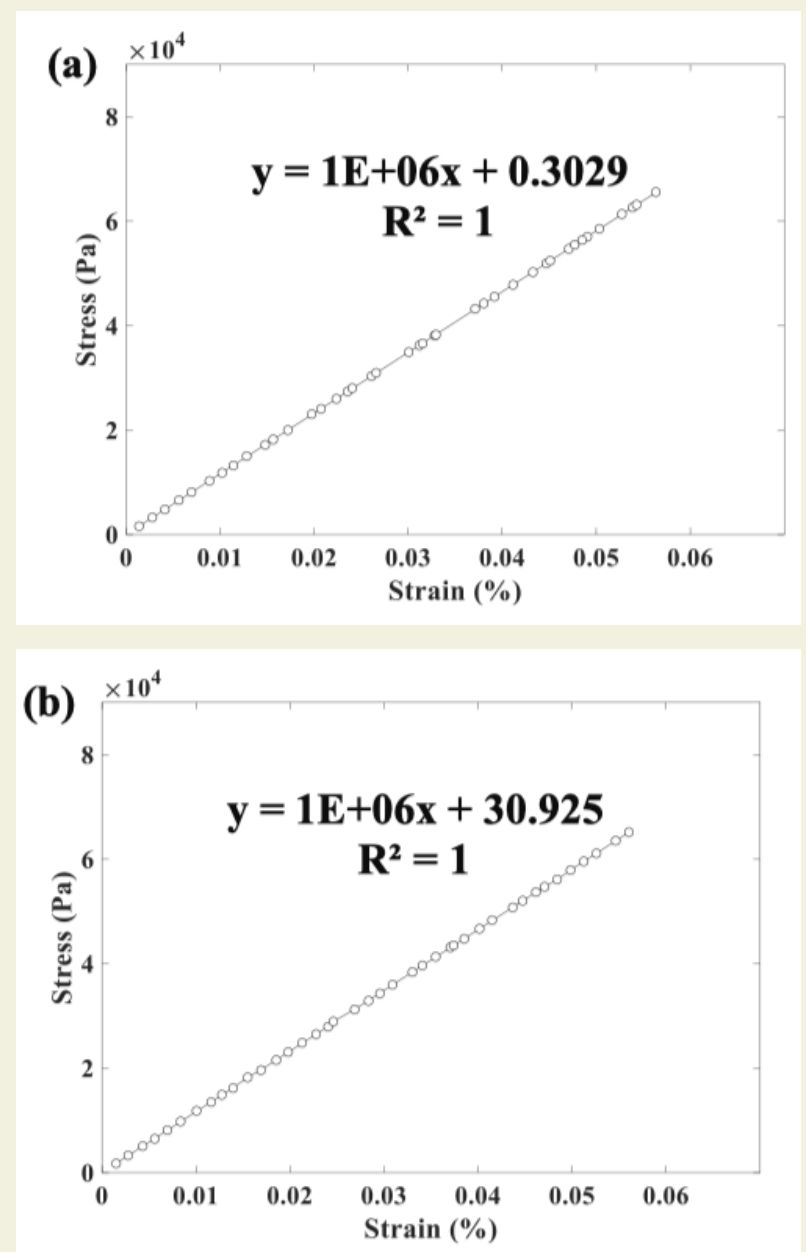

Figure 7. Stress-strain response of the biceps brachii, a) one-directional b) bidirectional.

al loading, while $65172 \mathrm{~Pa}$ stress occurs in bidirectional loading. Maximum strains were $0.0563 \%$ and $0.0560 \%$, respectively.

In this study, the mechanical behavior of the biceps brachii muscle fiber under different static loads was investigated. The analysis was performed according to two different one-way and two-way positions. According to the results, it could be seen that the amount of muscle fiber displacement in bilateral loading was significantly higher. This indicates that greater deformation and muscle strength will occur if force was transferred from the proximal and distal tendons of the biceps brachii. In addition, the stress and strain behavior and displacement increase linearly with the applied static load. These results were similar to those of Siebert et al [9]. The obtained results could be used to evaluate the exercise movements that cause isometric contraction of the muscles. Considering the results of this study, it could be said that a significant deformation might occur in the muscles, especially in resistance exercises performed using body weight against external load (for example, pull-up movement by attaching a plate to the waist area). While two-way loading may be detrimental to exercise, it could be considered an advan- tage, especially in post-stroke rehabilitation techniques. In cases of muscle and nerve damage such as stroke, muscle activation could be increased by bidirectional loading on the muscles. This might increase the effectiveness of exercise and shorten the rehabilitation processes.

\section{Conclusion}

The movement system of the human body, on the one hand, is a living and biological structure and depends on the laws of living things, on the other hand, it has a mechanical structure influenced by mechanical laws. The expansion and contraction of the biceps brachii changes the position of the elbow joint and causes it to move. Therefore, in this study, forces between $2.5 \mathrm{~N}$ and $100 \mathrm{~N}$ by $2.5 \mathrm{~N}$ steps were applied to investigate the mechanical behavior of the biceps brachii. Consequently, a linear correlation between force and displacement, strain and stress was obtained. In future studies, the electrical behavior of the biceps brachii and the role and importance of each of the above-mentioned structures will be examined in detail.

\section{Acknowledgements}

This work was supported by Research Fund of the Sakarya University of Applied Sciences. Project Number: 2021-01-04-055.

\section{References}

[1] Pandy, M. G., Barr, R. E. (2004). Biomechanics of the musculoskeletal system. Standard Handbook of Biomedical Engineering \& Design. McGRAW-HILL.

[2] Nordin, M., Frankel, V. H. (2001). Basic Biomechanics of the Musculoskeletal System. Lippincott Williams \& Wilkins, USA.

[3] Huxley, H. E. (1969). The mechanism of muscular contraction. Science, 164(3886): 1356-1366.

[4] Huxley, A. F. (1974). Muscular contraction. The Journal of Physiology, 243(1): 1.

[5] Hatze, H. (1981). Myocybernetic Control Models of Skeletal Muscle. Characteristics and Applications, University of South Africa.

[6] Riek, S., Chapman, A. E., Milner, T. (1999). A simulation of muscle force and internal kinematics of extensor carpi radialis brevis during backhand tennis stroke: implications for injury. Clinical Biomechanics, 14(7): 477-483.

[7] Stojanovic, B., Kojic, M., Rosic, M., Tsui, C. P., Tang, C. Y. (2007). An extension of Hill's three-component model to include different fibre types in finite element modelling of muscle. International Journal for Numerical Methods in Engineering, 71(7): 801-817.

[8] Tang, C. Y., Tsui, C. P., Stojanovic, B., Kojic, M. (2007). Finite element modelling of skeletal muscles coupled with fatigue. International Journal of Mechanical Sciences, 49(10): 1179-1191.

[9] Siebert, T., Stutzig, N., Rode, C. (2018). A hill-type muscle model expansion accounting for effects of varying trans- 
verse muscle load. Journal of Biomechanics, 66: 57-62.

[10] Wittek, A., Kajzer, J., Haug, E. (2000). Hill-type muscle model for analysis of mechanical effect of muscle tension on the human body response in a car collision using an explicit finite element code. JSME International Journal Series A Solid Mechanics and Material Engineering, 43(1): 8-18.

[11] Audu, M. L., Davy, D. T. (1985). The influence of muscle model complexity in musculoskeletal motion modeling. Journal of Biomechanical Engineering, 107(2): 147-157.

[12] Pandy, M. G., Zajac, F. E., Sim, E., Levine, W. S. (1990). An optimal control model for maximum-height human jumping. Journal of Biomechanics, 23(12): 1185-1198.

[13] Giat, Y., Mizrahi, J., Levine, W. S., Chen, J. (1994). Simulation of distal tendon transfer of the biceps brachii and the brachialis muscles. Journal of Biomechanics, 27(8): 1005-1014.

[14] Neptune, R. R., Sasaki, K., Kautz, S. A. (2008). The effect of walking speed on muscle function and mechanical energetics. Gait \& Posture, 28(1): 135-143.

[15] Maas, R., Siebert, T.,Leyendecker, S. (2012). On the relevance of structure preservation to simulations of muscle actuated movements. Biomechanics and Modeling in Mechanobiology, 11(3-4): 543-556.

[16] Rupp, T. K., Ehlers, W., Karajan, N., Günther, M., Schmitt, S. (2015). A forward dynamics simulation of human lumbar spine flexion predicting the load sharing of intervertebral discs, ligaments, and muscles. Biomechanics and Modeling in Mechanobiology, 14(5): 1081-1105.

[17] Bernabei, M., van Dieën, J. H., Baan, G. C., Maas, H. (2015). Significant mechanical interactions at physiological lengths and relative positions of rat plantar flexors. Journal of Applied Physiology, 118(4): 427-436.

[18] Reinhardt, L., Siebert, T., Leichsenring, K., Blickhan, R., Böl, M. (2016). Intermuscular pressure between synergistic muscles correlates with muscle force. Journal of Experimental Biology, 219(15): 2311-2319.

[19] Delp, S. L., Loan, J. P., Hoy, M. G., Zajac, F. E., Topp, E. L., Rosen, J. M. (1990). An interactive graphics-based model of the lower extremity to study orthopaedic surgical procedures. IEEE Transactions on Biomedical Engineering, 37(8): 757-767.

[20] Edman, K. A. (1988). Double-hyperbolic force-velocity relation in frog muscle fibres. The Journal of Physiology, 404(1): 301-321.

[21] Gordon, A. M., Huxley, A. F., Julian, F. J. (1966). The variation in isometric tension with sarcomere length in vertebrate muscle fibres. The Journal of Physiology, 184(1): 170-192.

[22] Katz, B. (1939). The relation between force and speed in muscular contraction. The Journal of Physiology, 96(1): 4564.

[23] Lieber, R. L., Boakes, J. L. (1988). Muscle force and moment arm contributions to torque production in frog hindlimb. American Journal of Physiology-Cell Physiology, 254(6): 769-772.

[24] Scott, S. H., Brown, I. E., Loeb, G. E. (1996). Mechanics of feline soleus: I. Effect of fascicle length and velocity on force output. Journal of Muscle Research \& Cell Motility, 17(2): 207-219.

[25] Lieber, R. L. (1993). Skeletal Muscle Architecture: Implica- tions for Muscle Function and Surgical Tendon Transfer. Journal of Hand Therapy, 6(2): 105-113.

[26] Shue, G. H., Crago, P. E. (1998). Muscle-tendon model with length history-dependent activation-velocity coupling. Annals of Biomedical Engineering, 26(3): 369-380.

[27] Van Donkelaar, C. C., Willems, P. J. B., Muijtjens, A. M. M., Drost, M. R. (1999). Skeletal muscle transverse strain during isometric contraction at different lengths. Journal of Biomechanics, 32(8): 755-762.

[28] Stäubli, H. U., Schatzmann, L., Brunner, P., Rincón, L., Nolte, L. P. (1999). Mechanical tensile properties of the quadriceps tendon and patellar ligament in young adults. The American Journal of Sports Medicine, 27(1): 27-34.

[29] Holzbaur, K. R., Murray, W. M., Delp, S. L. (2005). A model of the upper extremity for simulating musculoskeletal surgery and analyzing neuromuscular control. Annals of Biomedical Engineering, 33(6): 829-840.

[30] Martinek, J., Stickler, Y., Reichel, M., Mayr, W., Rattay, F. (2008). A Novel Approach to Simulate Hodgkin-Huxleylike Excitation With COMSOL Multiphysics. Artificial Organs, 32(8): 614-619.

[31] Kocbach, J., Folgero, K., Mohn, L., Brix, O. (2011). A simulation approach to optimizing performance of equipment for thermostimulation of muscle tissue using COMSOL multiphysics. Biophysics and Bioengineering Letters, 4(2): 9-33.

[32] Carbone, V., van der Krogt, M. M., Koopman, H. F., Verdonschot, N. (2016). Sensitivity of subject-specific models to Hill muscle-tendon model parameters in simulations of gait. Journal of Biomechanics, 49(9): 1953-1960.

[33] Esmaeili, J., Maleki, A. (2020). Muscle coordination analysis by time-varying muscle synergy extraction during cycling across various mechanical conditions. Biocybernetics and Biomedical Engineering, 40(1): 90-99. 